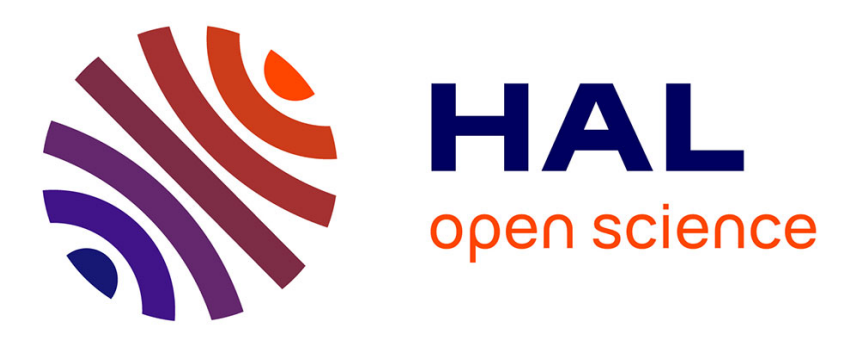

\title{
Intraband transition in self-doped narrow band gap colloidal quantum dots
}

Bertille Martinez, Clément Livache, Adrien Robin, Hervé Cruguel, Sébastien Royer, Xiangzhen Xu, Hervé Aubin, Sandrine Ithurria, Emmanuel Lhuillier

\section{- To cite this version:}

Bertille Martinez, Clément Livache, Adrien Robin, Hervé Cruguel, Sébastien Royer, et al.. Intraband transition in self-doped narrow band gap colloidal quantum dots. SPIE phpotonic west, Jan 2017, San Fransisco, United States. 10.1117/12.2250155 . hal-01476526

\section{HAL Id: hal-01476526 https://hal.science/hal-01476526}

Submitted on 25 Aug 2020

HAL is a multi-disciplinary open access archive for the deposit and dissemination of scientific research documents, whether they are published or not. The documents may come from teaching and research institutions in France or abroad, or from public or private research centers.
L'archive ouverte pluridisciplinaire HAL, est destinée au dépôt et à la diffusion de documents scientifiques de niveau recherche, publiés ou non, émanant des établissements d'enseignement et de recherche français ou étrangers, des laboratoires publics ou privés. 


\title{
Intraband transition in self-doped narrow band gap colloidal quantum dots
}

\author{
Bertille Martinez ${ }^{1}$, Clément Livache, ${ }^{1,2}$ Adrien Robin, ${ }^{2}$ Herve Cruguel, ${ }^{1}$ Sebastien Royer, ${ }^{1}$ \\ Xiang Zhen $\mathrm{Xu}^{2}$, Herve Aubin ${ }^{2}$, Sandrine Ithurria ${ }^{2}$, Emmanuel Lhuillier ${ }^{1 *}$ \\ ${ }^{1}$ Sorbonne Universités, UPMC Univ Paris 06, Institut des Nanosciences de Paris, UPMC- \\ CNRS UMR 7588, 4 place Jussieu, boîte courrier 840, 75252 Paris cedex 05, France \\ ${ }^{2}$ Laboratoire de Physique et d'Étude des Matériaux, PSL Research University, CNRS UMR \\ 8213, Sorbonne Universités UPMC Univ Paris 06, ESPCI ParisTech, 10 rue Vauquelin, \\ 75005 Paris, France.
}

\begin{abstract}
In this article we discuss the infrared properties of self-doped nanocrystals and in particular the case of $\mathrm{HgSe}$. HgSe colloidal quantum dots have recently been reported for their tunable optical features all over the mid infrared from 3 to $20 \mu \mathrm{m}$. Their optical absorption is a combination of interband absorption at high energy and intraband absorption at low energy. The latter results from the self-doped character of $\mathrm{HgSe}$. The origin of this self-doping is also discussed. We demonstrated that the doping results from the combination of the narrow band gap and high work function of $\mathrm{HgSe}$, which leads to a reduction of the CQD by the water in the environment. In addition, we demonstrated that the doping density can be tuned over an order of magnitude thanks to the control of the capping ligands.
\end{abstract}

KEYWORDS nanocrystal, narrow band gap, mid-infrared, self-doping.

\section{INTRODUCTION}

The use of colloidal quantum dots (CQD) for optoelectronics [1] has so far been mostly focused on the absorption of the near infrared tail of the sun emission for solar cell application. In this sense, lead chalcogenides have in particular obtained some success with efficiency now reaching $10 \%[2,3]$. The mid infrared range of wavelength remains far less investigated mostly because narrower band gap materials [4] are necessary to push the optical features toward smaller energy transitions. To achieve low energy transition, an alternative path to narrow band gap interband transition is the use of intraband transition. In this case, electronic transition occurs between conduction band states. This approach has been very successful for epitaxially grown III-V semiconductor heterostructures. Typically, a doped quantum well of GaAs is used in multi-quantum well heterostructure to design Quantum Well Infrared Phototodetectors (QWIP [5,6]). In nanocrystals, the doping remains far less controlled than epitaxially grown semiconductor. Consequently, physical charging of the CQD through optical pumping [7] or electrochemical charging [8] allows injecting charges and observing intraband absorption. More recently, the doping of silicon [9] and oxide [10] nanoparticles has been demonstrated and used to induce electrochomic properties.

Self-doped CQD have only been reported in 2014. In this case, CQD of $\mathrm{HgS}[11,12]$ and $\mathrm{HgSe}[13,14]$ have been obtained under a doped stable form while no intentional step to induce doping was conducted. These new materials raise great interest for infrared photodetection, because these CQD combine absorption in the mid infrared with photoconductive properties. In this paper, we discuss the recent progresses obtained on $\mathrm{HgSe}$ with tunable optical features all over the mid infrared from 3 to $20 \mu \mathrm{m}$. 




Figure 1 TEM image of small (a) and large (b) HgSe colloidal quantum dots. The insets are high resolution images. c. Image of a large scale batch leading to more than $10 \mathrm{~g}$ of nanocrystals, adapted from [14].

\section{DISCUSSION}

So far, most of the effort for the synthesis of mid infrared CQD [15] has been focused on $\mathrm{HgTe}$, [16,17] because of its proximity with the $\mathrm{HgCdTe}$ alloy. $\mathrm{HgSe}$ has been far less investigated, in spite of the fact that the bulk band structure is very close to the one of $\mathrm{HgTe}[18,19]$. The two materials are semimetals with inverted band structures. In the case of $\mathrm{HgSe}$, most of the past reports have been focused on the synthesis of small nanoparticles which present absorption in the near infrared [20,21,22 ]. Recently, the Guyot-Sionnest's group pointed out by that $\mathrm{HgS}$ [11] and $\mathrm{HgSe}$ [13] can be synthetized under a doped form, which exhibits an intraband feature between 300 and $2000 \mathrm{~cm}^{-1}$, see Figure 2a.
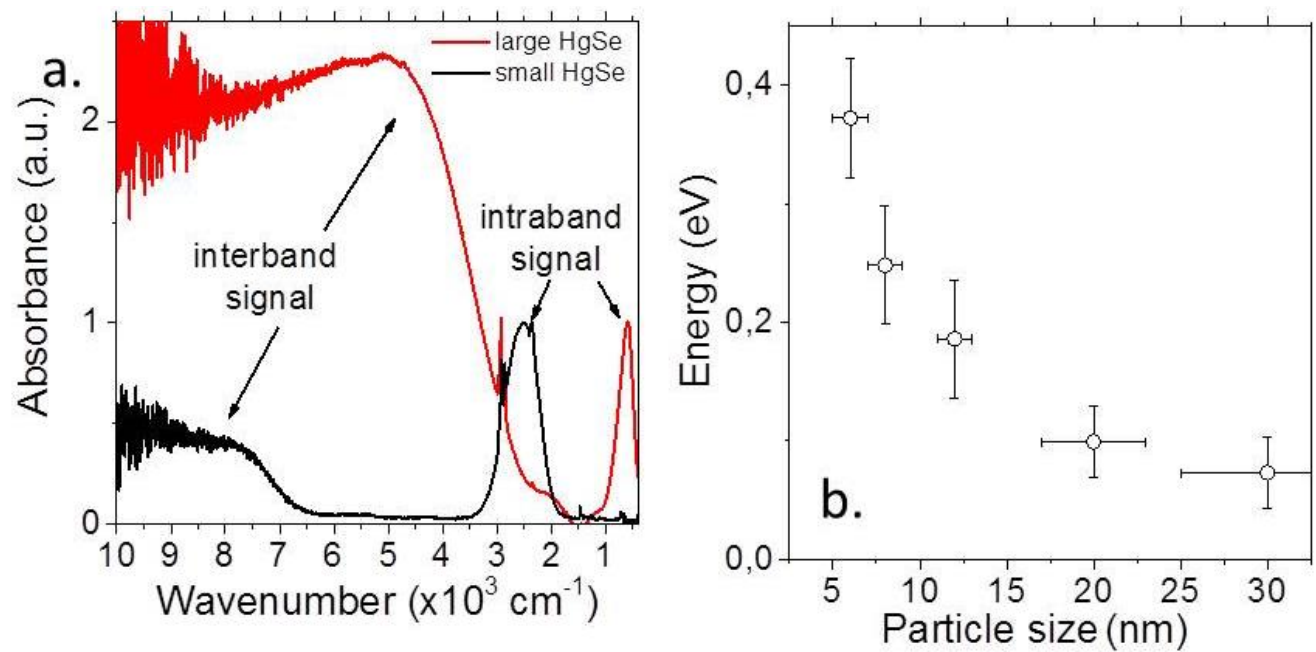

Figure 2 a. Absorption spectrum of small and large $\mathrm{HgSe} C Q D$, adapted from [14]. b. Energy of the intraband transition of $\mathrm{HgSe} C Q D$ as a function of the nanocrystal size (diameter).

We suggested an updated synthesis for HgSe which allows a large tuning of the size from 3 to $50 \mathrm{~nm}$, see Figure $1 \mathrm{a}$ and $\mathrm{b}$. In a first step, a complex of mercury oleate oleylamine is prepared. Then, depending on the targeted size of CQD, two selenium precursors can be used: selenium complexed with trioctylphosphine for small $\mathrm{HgSe} C \mathrm{CQ}$ or $\mathrm{SeS}_{2}$ diluted in oleylamine for large $\mathrm{HgSe} \mathrm{CQD}$. In addition, a complementary control of the CQD size can also result from temperature $\left(60-120^{\circ} \mathrm{C}\right)$ and reaction duration $(1 \mathrm{~min}$ to $30 \mathrm{~min})$. Large scale synthesis up to $10 \mathrm{~g}$ has also been demonstrated, see Figure 1c. The obtained material shows two characteristic optical features. At high 
energy, a broad feature corresponds to the interband transition, see Figure 2a. At low energy a second contribution relative to the intraband transition appears under a peak form. Thanks to the wide tunability of size obtained for the synthesis of $\mathrm{HgSe}$, the mid infrared peak energy can be tuned from $3 \mu \mathrm{m}(400 \mathrm{meV})$ up to $20 \mu \mathrm{m}(50 \mathrm{meV})$, see Figure $2 b$.

Since the intraband absorption is the signature of the doped character of the CQD, a deeper understanding of the doping origin is mandatory. It has been demonstrated that the relative magnitude of the interband and intraband absorption strongly depend on the nanocrystal surface chemistry. Typically, the growth of a CdS shell tends to reduce the intraband absorption [23], see Figure 3a. Similarly, changing the initial long thiol chain for shorter ligands also strongly affects the electronic spectrum, see Figure $3 \mathrm{~b}$. The relative weight of the intraband absorption decreases and the $1 S_{h}$ to $1 S_{\mathrm{e}}$ transition, which is initially bleached, appears. This change of the spectrum while changing surface ligand is stronger for smaller CQD, which suggests that doping is not a surface effect. Since we consider a bulk origin of doping, we integrate two main facts: (i) mercury chalcogenides have a large workfunction and (ii) even under a CQD form, they show a limited confinement. As a result, the conduction band of the $\mathrm{HgSe} C Q D$ can be deeper (versus vacuum) than the $\mathrm{O}_{2} / \mathrm{H}_{2} \mathrm{O}$ redox couple $(5.7 \mathrm{eV}$ versus vacuum). The reduction of the CQD by water becomes possible and the stable form of the CQD is negatively charged [24].
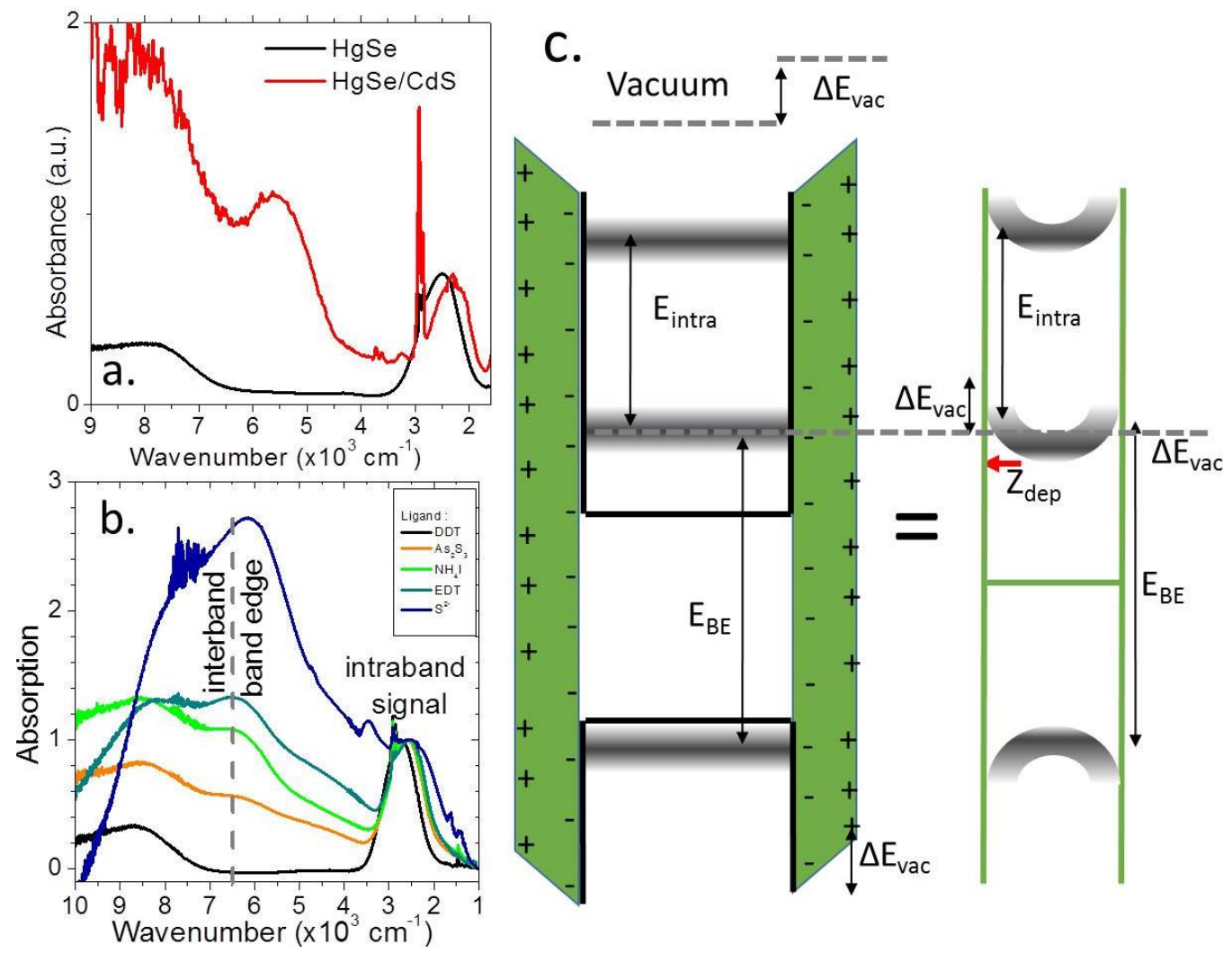

Figure 3 a. Absorption spectrum of $\mathrm{HgSe}$ and $\mathrm{HgSe} / \mathrm{CdS} C Q D$. b. Absorption spectrum of $\mathrm{HgSe}$ CQD capped with different ligands. c. Scheme of the electronic structure of $\mathrm{HgSe} C Q D$ once functionalized by dipoles. EBE corresponds to the energy of the band edge transition. Adapted from ref [24].

When ligands get exchanged, dipoles are added on the surface. Since these dipoles present a negative charge at the CQD surface, the CQD screen this charge by bringing positive charges at the surface which band the electronic levels and decreases the overall negative doping, see Figure $3 \mathrm{c}$. We can relate the band bending magnitude $\Delta E_{v a c}$ to the density of dipole $(N)$ and their magnitude $(\mu)$ via the relation $\Delta E_{\text {vac }}=-N \frac{\mu_{\perp}(\text { ligand })}{\varepsilon_{0} \varepsilon_{\text {ligand }}}, \varepsilon_{0} \varepsilon_{\text {ligand }}$ being the product of the vacuum permittivity by the medium dielectric constant. Small CQD show a large confinement, which shift their band above the $\mathrm{O}_{2} / \mathrm{H}_{2} \mathrm{O}$ and no reduction is observed for the smallest nanocrystals, see Figure $4 \mathrm{a}$. On the other hand, this reduction process is favored in large, poorly confined CQD, see Figure $4 \mathrm{~b}$. The larger the 
CQD, the higher the doping. We can also quantify the impact of the ligand exchange on the CQD population, see Figure $4 \mathrm{c}$, and we observe a drop of the carrier density by a factor 10 while the capping ligand switch from dodecanthiol to $\mathrm{S}^{2-}$.
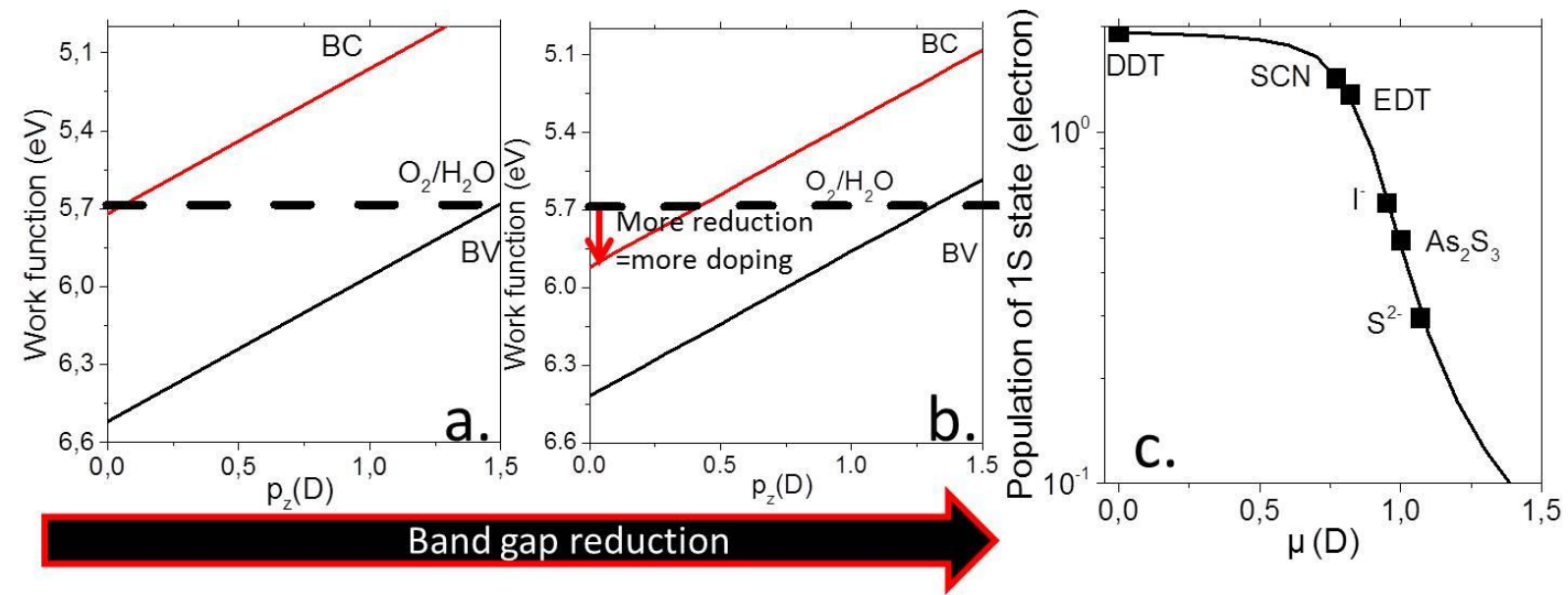

Figure 4 Electronic structure (conduction and valence band) of a. small b. large HgSe CQD as a function of the surface dipole magnitude. c. Population of the 1 s state as a function of the dipole magnitude. The relative population associated with different capping ligand has been added to estimate the associated dipole magnitude, adapted from [24].

We can also find evidence for doping in the transport data. To do so, we first build an electrolytic transistor, which channel is made of highly monodisperse HgSe CQD. The CQD are capped with ionic passivation made of $\mathrm{As}_{2} \mathrm{~S}_{3}$ because of the material transparency in the mid infrared $[25,26]$ and the fairly high carrier mobility otained. Iongel electrolyte is chosen because of its inherent large gate capacitance, which allows achieving carrier density of several carriers per CQD [27]. In addition, they offer air operability of the transistor and are able to charge thick films of nanocrystals, which is very convenient to design phototransistors [28].
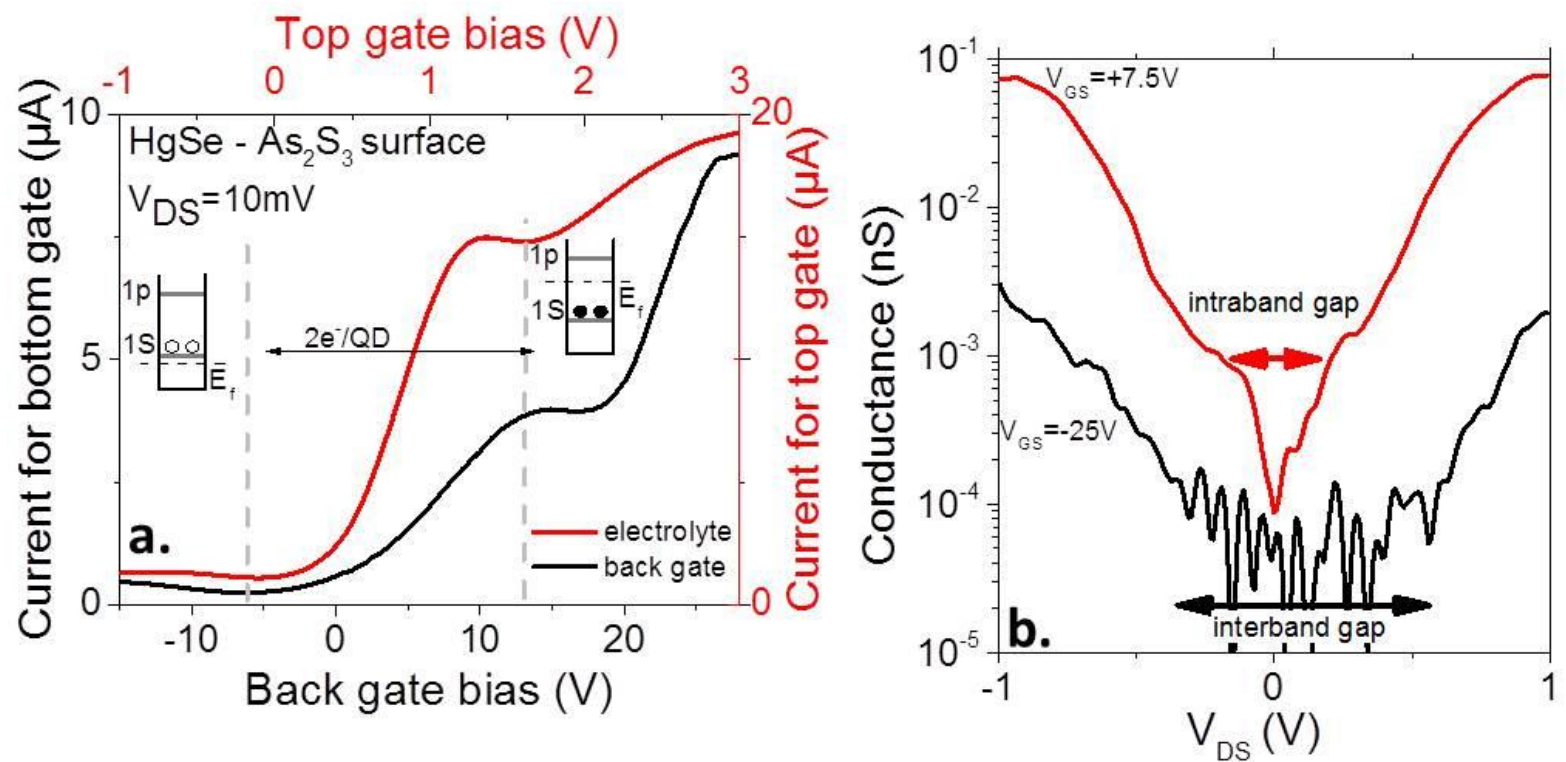

Figure 5 a. Transfer curve (drain current as a function of gate bias) for a dual gated transistor, which channel is made of HgSe $\mathrm{CQD}$, adapted from [14]. The gate can be a $\mathrm{SiO}_{2}$ dielectric layer or an electrolyte. $b$. Tunnel conductance of a single $\mathrm{HgSe}$ $C Q D$, , adapted from [31]. 
The transistor transfer curve (drain current versus gate bias, see Figure 5a) is typical of an $n$-type material, which confirms that the material is electron rich. In addition, the curve is non monotonic, as it shows a local minimum. The latter is the signature of the Pauli blockade [29]. Once two electrons per CQD are injected, the 1s state is filled and transport occurs through the $1 \mathrm{p}$ state. This requires some thermal activation and leads to a drop of mobility.

If transport is conducted at the single particle scale, in an on chip tunnel spectroscopy configuration $\left[{ }^{30}\right]$, we also observe a signature of the doping ${ }^{31}$. The tunnel spectrum measured under gate biases are shown in Figure 5a. Under hole injection $\left(\mathrm{V}_{\mathrm{GS}}<0\right)$, the measured band gap is large, which, assuming an arm leaver of $1 / 2$, corresponds to a excitonic gap of $0.8 \mathrm{eV}$, in good agreement with the optical interband gap. Once electrons are injected in the CQD $\left(\mathrm{V}_{\mathrm{GS}}>0\right)$, the tunnel band gap shrinks down to $0.4 \mathrm{eV}$, which is now consistent with the intraband gap. Thus tunnel spectroscopy confirms that doping exists even at the single particle level.

\section{CONCLUSION}

We investigate the optical and transport properties of HgSe CDQ. These CQD present self-doping leading to an additional optical feature in the mid infrared range. This peak results from the reduction of the CQD by its environment due to the high work function of the material and limited confinement. The doping level can be further tuned by changing the capping dipole. The doped character of the CQD is also confirmed by the transport measurement which presents Pauli blockade, as the 1s state is fully filled. Moreover, the tunnel band gap is strongly modified, when the Fermi level is switched from the interband gap to the intraband gap.

\section{ACKNOWLEDGEMENTS}

This work was supported by French state funds managed by the ANR within the Investissements d'Avenir program under reference ANR-11-IDEX-0004-02, and more specifically within the framework of the Cluster of Excellence MATISSE led by Sorbonne Universités. SI thanks the ANR for funding through the Nanodose grant.

\section{REFERENCES}

[1] D.V. Talapin, J.S. Lee,. M. Kovalenko, E. Shevchenko, "Prospects of Colloidal Nanocrystals for Electronic and Optoelectronic Applications", Chem. Rev. 110, 389 (2010).

[2]C. H. M.Chuang, P. R.Brown V. Bulovic, M. G. Bawendi " Improved performance and stability in quantum dot solar cells through band alignment engineering » Nature Materials. 13, 796 (2014).

[3] X. Lan, O. Voznyy, F. P. García de Arquer, M. Liu, J. Xu, A. H. Proppe,G. Walters, F. Fan, H. Tan, M. Liu, Z. Yang, S. Hoogland, and E. H. Sargent, "10.6\% Certified Colloidal Quantum Dot Solar Cells via Solvent-Polarity-Engineered Halide Passivation", Nano Lett., 16, 4630 (2016)

[4]S. V. Kershaw, A. S. Susha and A. L. Rogach, "Narrow bandgap colloidal metal chalcogenide quantum dots: synthetic methods, heterostructures, assemblies, electronic and infrared optical properties," Chem. Soc. Rev. 42, 3033 (2013).

[5]H. Schneider and H.C. Liu, Quantum well infrared photodetectors, Physics and applications (Springer, Heidelberg, 2006). [6]A. Rogalski, J. Antoszewski, L. Faraone, "Third-generation infrared photodetector arrays", J. Appl. Phys. 105, 091101 (2009)

[7]P Guyot-Sionnest, MA Hines "Intraband transitions in semiconductor nanocrystals,",Appl. Phys. lett. 72, 686 (1999).

[8]C. Wang, M. Shim, P. Guyot-Sionnest, Electrochromic nanocrystal quantum dots, Science 291, 2390 (2001).

[9]N.J. Kramer, K. S. Schramke and U. R. Kortshagen, "Plasmonic Properties of Silicon Nanocrystals Doped with Boron and Phosphorus," Nano Lett. 15,5597 (2015)

[10]E. L. Runnerstrom, A. Llordes, S. D. Lounis, D. J. Milliron. "Nanostructured electrochromic smart windows: traditional materials and NIR-selective plasmonic nanocrystals," Chem. Comm. 50, 10555 (2014).

[11]K. S. Jeong, Z. Deng, S. Keuleyan, H. Liu, P. Guyot-Sionnest “Air-Stable n-Doped Colloidal HgS Quantum Dots”, J. Phys. Chem. Lett. 5, 1139 (2014).

[12]B. Yoon, J. Jeong, K. S. Jeong, "Higher Quantum State Transitions in Colloidal Quantum Dot with Heavy Electron Doping," J Phys. Chem C 120, 22062 (2016)

[13]Deng Z., Jeong K. S., Guyot-Sionnest P. “Colloidal Quantum Dots Intraband Photodetectors”, ACS Nano 8, 11707 (2014) 
[14]E. Lhuillier, M. Scarafagio, P. Hease, B. Nadal, H. Aubin, X. Z. Xu, N. Lequeux, G. Patriache, S. Ithurria and B. Dubertret, "Infrared photo-detection based on colloidal quantum-dot films with high mobility and optical absorption up to the THz" Nano Lett 16, 1282 (2016).

[15]E. Lhuillier, S. Keuleyan, H. Liu, P. Guyot-Sionnest “ Mid-IR Colloidal Nanocrystals”, Chem Mat. 25, 1272 (2013)

[16]S. Keuleyan, E. Lhuillier, V. Brajuskovic, P. Guyot-Sionnest, « Mid-infrared HgTe colloidal quantum dot photodetectors”, Nat. Photon. 5, 489 (2011).

[17]M.V. Kovalenko, E. Kaufmann, D. Pachinger, J. Roither, M. Huber, J. Stangl, G. Hesser, F. Schaffler W. Heiss, "Colloidal HgTe nanocrystals with widely tunable narrow band gap energies: from telecommunications to molecular vibrations" J. Am. Chem. Soc. 128, 3516 (2008).

[18]G. Allan, C. Delerue, “Tight-binding calculations of the optical properties of HgTe nanocrystals," Phys. Rev. B 86, 165437 (2012)

[19]E. Lhuillier, S. Keuleyan, P. Guyot-Sionnest, “Optical properties of HgTe colloidal quantum dots”, Nanotechnology 23, 175705 (2012)

[20]H. Mirzai, M. N. Nordin, R.J. Curry, J.S. Bouillard, A.V. Zayats, M. Green, "The room temperature phosphine-free synthesis of near-infrared emitting HgSe quantum dots", J. Mater. Chem. C 2, 2107 (2014).

[21]P. Howes, M. Green, C. Johnston, A. Crossley, "Synthesis and shape control of mercury selenide (HgSe) quantum dots", J. Mater. Chem. 18, 3474 (2008).

[22]S. B. Qadri, M. Kuno, C.R. Feng, B.B. Rath, M. Yousuf, "High temperature structural studies of HgS and HgSe quantum dots", Appl. Phys. Lett. 83, 4011 (2003).

[23]G. Shen and P. Guyot-Sionnest, "HgS and HgS/CdS Colloidal Quantum Dots with Infrared Intraband Transitions and Emergence of a Surface Plasmon," J. Phys. Chem. C 120, 11744 (2016).

[24]A. Robin, C. Livache, S. Ithurria, E. Lacaze, B. Dubertret, E. Lhuillier, "Surface Control of Doping in self-doped Nanocrystals" ACS Appl. Mat. Interface 8, 27122 (2016).

[25] S. Yakunin, D.N. Dirin, L. Protesescu, M. Sytnyk, S. Tollabimazraehno, M. Humer, F. Hackl, T. Fromherz, M.I. Bodnarchuk, M.V. Kovalenko, W. Heiss, "High Infrared Photoconductivity in Films of Arsenic-Sulfide-Encapsulated LeadSulfide Nanocrystals", ACS Nano. 8, 12883 (2014).

[26]E. Lhuillier, S. Keuleyan, P. Zolotavin, P. , Guyot-Sionnest “ Mid-Infrared HgTe/As $2 \mathrm{~S}_{3}$ FETs and photodetectors” Adv. Mat. 25, 137 (2013).

[27]E. Lhuillier, S. Ithurria, A. Descamps-Mandine, T. Douillard, R. Castaing, X.Z. Xu, P-L. Taberna, P. Simon, H. Aubin, B. Dubertret, "Investigating the $\mathrm{n}$ and $\mathrm{p}$ type electrolytic charging of colloidal nanoplatelets", J Phys Chem C 119, 21795 (2015) [28]E. Lhuillier, A. Robin, S. Ithurria, H. Aubin, B. Dubertret, «Electrolyte gated colloidal nanoplatelets based phototransistor and its use for bicolor detection "Nano Lett. 14, 2715 (2014).

[29]D Yu, C Wang, P Guyot-Sionnest, «n-Type conducting CdSe nanocrystal solids », Science 300, 1277 (2003).

[30]H. Wang, E. Lhuillier, Q. Yu, A. Mottaghizadeh, C. Ulysse, A. Zimmers, A. Descamps-Mandine, B. Dubertret,H. Aubin, "Effects of electron-phonon interactions on the electron tunneling spectrum of PbS quantum dots" Phys. Rev. B 92, 041403 (2015).

[31]H. Wang, E. Lhuillier, Q. Yu, A. Zimmers, B. Dubertret, C. Ulysse and H. Aubin, "In-Vacuum projection of nanoparticles for on-chip tunnelling spectroscopy", submitted (2016). 